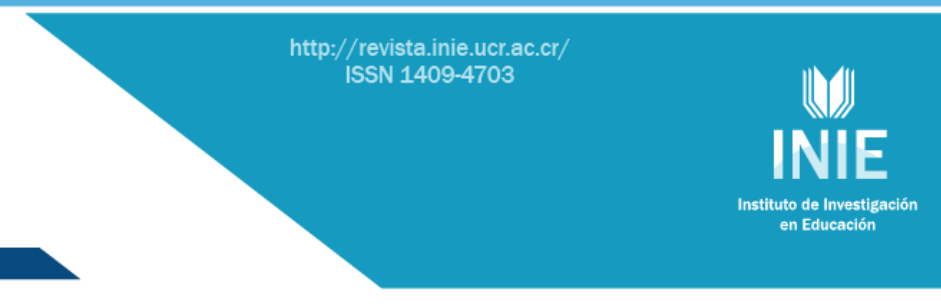

\title{
EL LENGUAJE ORDINARIO: LA CLAVE PARA EL APRENDIZAJE DE LAS MATEMÁTICAS BASADO EN PROBLEMAS \\ ORDINARY LANGUAGE: A KEY TO PROBLEM-BASED MATH LEARNING
}

\author{
Volumen 15, Número 1 \\ Enero - Abril \\ pp. 1-24
}

Este número se publicó el $1^{\circ}$ de enero de 2015

DOI: dx.doi.org/10.15517/aie.v15i1.17591

José Ángel García Retana

Revista indizada en REDALYC, $\underline{\text { SCIELO }}$

Revista distribuida en las bases de datos:

CATÁLOGO DE LATINDEX, IRESIE, CLASE, DIALNET, DOAJ, E-REVIST@S, SHERPA/ROMEO, QUALIS, MIAR

Revista registrada en los directorios:

ULRICH'S, $\underline{\text { REDIEE}}, \underline{\text { RINACE}}, \underline{\text { OEI }}$ MAESTROTECA, PREAL, $\underline{\text { CLACSO }}$ 


\title{
EL LENGUAJE ORDINARIO: LA CLAVE PARA EL APRENDIZAJE DE LAS MATEMÁTICAS BASADO EN PROBLEMAS \\ ORDINARY LANGUAGE: A KEY TO PROBLEM-BASED MATH LEARNING
}

\author{
José Ángel García Retana ${ }^{1}$
}

\begin{abstract}
Resumen: En el año 2012 el Ministerio de Educación Pública de Costa Rica, planteó una nueva propuesta de educación matemática para responder a las exigencias sociales y económicas actuales. Esta propuesta se fundamenta en el aprendizaje basado en problemas (ABP) como estrategia metodológica. En el caso del aprendizaje de las matemáticas, tal propuesta demanda considerar la relación que existe entre el lenguaje ordinario y el lenguaje matemático, por cuanto el primero es central en el proceso educativo. Este tipo de aprendizaje se debe conceptualizar en su doble función de herramienta, es decir, para resolver problemas, y como disciplina, dado que el lenguaje matemático permite representar los conceptos que trata, al menos de dos maneras diferentes, la semántica y la gráfico-visual, los cuales en gran medida son determinados por el lenguaje ordinario. Así, el lenguaje ordinario y su campo semántico constituyen el eje transversal para el aprendizaje de esta estrategia metodológica.
\end{abstract}

Palabras clave: LENGUAJE ORDINARIO, MATEMÁTICAS, PROBLEMAS, APRENDIZAJE

\begin{abstract}
In the year 2012, the Ministry of Public Education of Costa Rica recommended a new proposal for the teaching of mathematics in order to respond to current social and economic requirements. This proposal is grounded on problem-based learning (PBL) as a methodological learning strategy. For the learning of mathematics, such a proposal demands for a co-existing relationship between the ordinary language and the mathematical language, considering ordinary language as the central aspect in the educational process. This type of learning should be conceptualized in its two-way function; as a resource to solve problems, and as a discipline, to represent concepts, based on the premise that the mathematical language allows concepts to be represented in two different ways: semantically and graphic-visually. In fact, these concept representations are largely determined by the ordinary language as well. Thus, ordinary language and its semantic field constitute the cross-curricular axis for the learning of this methodological strategy.
\end{abstract}

Key Words: ORDINARY LANGUAGE, MATHEMATICS, PROBLEMS, LEARNING

\footnotetext{
${ }^{1}$ Profesor en Secundaria y en la Sede Guanacaste, Universidad de Costa Rica. Licenciado en la Enseñanza de la Matemática, Universidad de Costa Rica. Reside desde 1996 en Liberia, Guanacaste donde ha desarrollado su labor docente.
}

Dirección electrónica: jose.garcia@ucr.ac.cr

Ensayo recibido: 9 de mayo, 2014

Enviado a corrección: 29 de setiembre, 2014

Aprobado: $1^{\circ}$ de diciembre, 2014 


\section{Introducción}

El 21 de Mayo del año 2012, el Consejo Superior de Educación de Costa Rica aprobó la implementación de un nuevo programa para el aprendizaje y enseñanza de las matemáticas, tanto para la Educación General Básica como para el Ciclo Diversificado. El objetivo fundamental de este programa es procurar poner el proceso de aprendizajeenseñanza de las matemáticas a la altura de las demandas y lineamientos que para tal efecto, plantea la Organización para la Cooperación y el Desarrollo (OCDE), organización a la que el gobierno de Costa Rica mostró su aspiración, en ese momento, por incorporarse como parte de la inserción del país en la economía globalizada actual.

Este programa, al menos en términos del discurso, parte de los planteamientos del socio constructivismo, así como de la solución de problemas como parte del Aprendizaje Basado en Problemas (ABP) en calidad de recurso metodológico, a lo que propone adjuntar un uso prudente de las tecnologías de la información y la comunicación (tecnologías digitales). De este modo, se pretende, de manera gradual y racional, generar una cultura matemática donde los procesos cognitivos y metacognitivos, ligados con la solución de problemas, estén por encima del uso indiscriminado de la tecnología. El optar por el ABP no es casualidad, sino porque se consideró que "La resolución de problemas corresponde a la necesidad de asumir estándares cuya conveniencia para la educación matemática ha sido ampliamente comprobada en la escala internacional" (Ministerio de Educación Pública, 2012, p. 17).

La base teórica del programa se encuentra, tanto en el Informe Delors (1996), en la conceptualización sobre "competencias" definida por la OCDE (OCDE, 2010), como en el Modelo Basado en Competencias (MBC). Esta propuesta educativa es estratégica si se toma en cuenta que para la economía globalizada es de suma importancia integrar la interacción entre los educandos, sus situaciones de aprendizaje y los contextos sociales, económicos y culturales en los cuales participan (Gómez, 1991). Se persigue con ello, una forma de comprender y hacer matemáticas, diferente a las propuestas durante el siglo XX, en la cual la actividad educativa se deberá centrar en el valor instrumental de la disciplina y en su capacidad para desarrollar habilidades, competencias y procesos capaces de potenciar el aprender a resolver problemas por parte de los educandos. Esto es, que el educando aprenda a actuar con criterio propio y pueda tomar decisiones, lo cual desde la perspectiva del MEP, significaría que "se impone una lógica del saber en contexto del aprender a aprender" (MEP, 2012, p. 14). 
Dado que el ABP es una propuesta que no ha sido ampliamente debatida o implementada en Costa Rica, su novedad amerita, para los efectos del presente ensayo, destacar los aspectos básicos y relevantes que la sitúan como estrategia metodológica, estos se muestras en la Figura 1, según el Instituto Tecnológico y de Estudios Superiores de Monterrey (ITESM, 2010):

Figura 1

Etapas de la metodología aprendizaje basado en problemas

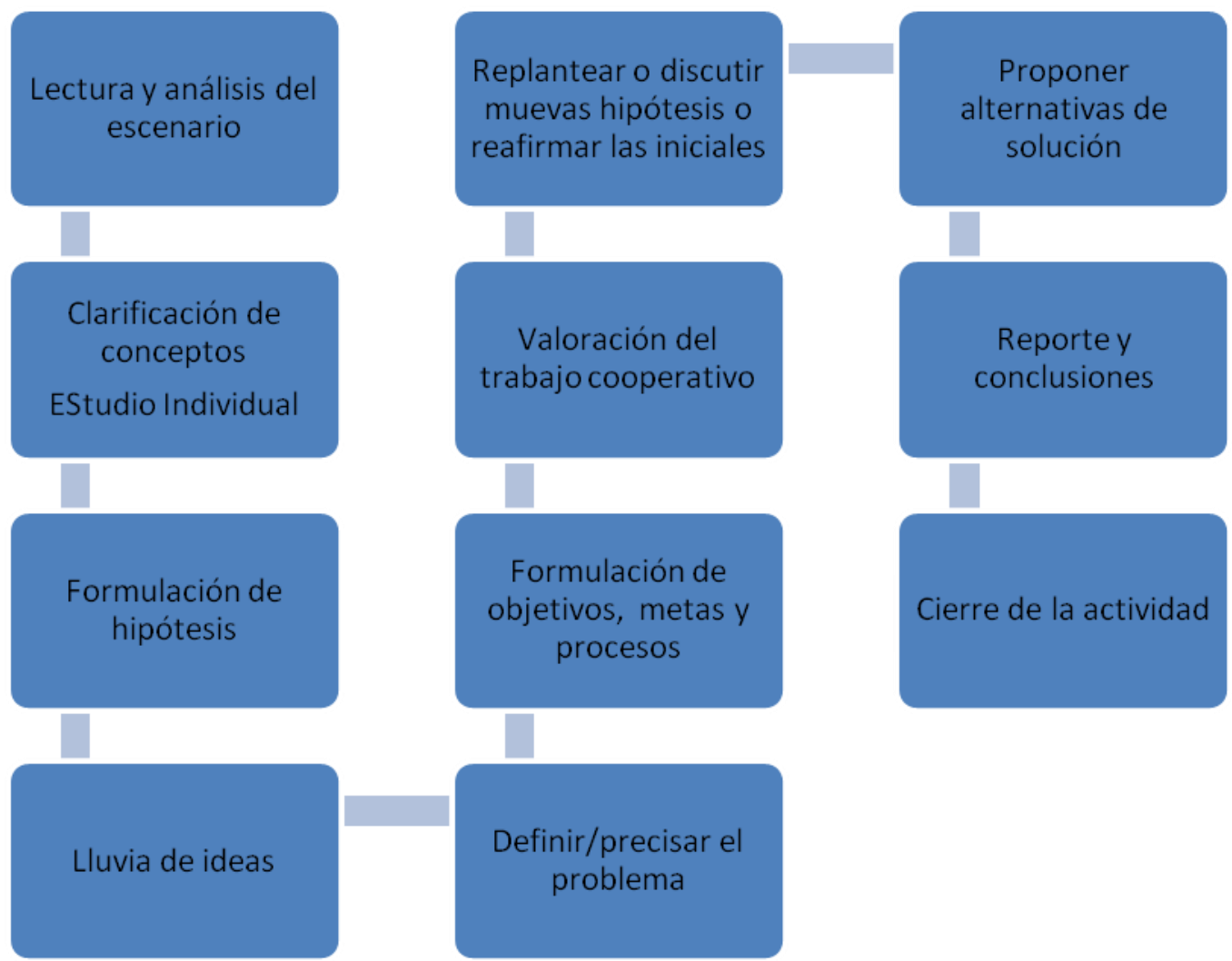

Fuente: Elaboración propia con base en Instituto Tecnológico de Monterrey (2010).

En el desarrollo de la implementación del ABP como proceso de aprendizaje, el profesor diseña los contenidos del problema, guía el proceso, retroalimenta, asegura que los alumnos no se pierdan en el camino y evalúa. El alumno, por su parte, debe asumir la responsabilidad de investigar, organizar e intercambiar la cantidad y calidad de la información necesaria para definir el problema planteado en el escenario. Por último, el colectivo debe administrar el trabajo individual y el colaborativo para procurar crear 
soluciones pertinentes, hacer un uso eficiente de los recursos y el tiempo de todos los integrantes del equipo, para poder generar propuestas de solución.

Los requisitos para un buen desarrollo de la técnica se enuncian a continuación:

- Revisar minuciosamente el escenario, el cual provee una situación problemática que requiere del participante conocimientos y habilidades específicas para ser resuelta.

- Seguir todas las etapas y actividades de la metodología, aun cuando parezca que no es necesario realizarlas todas. Cada una de ellas tiene un propósito y facilita el camino hacia la solución del problema.

- Definir y asumir con responsabilidad las tareas de cada integrante del equipo. Esto facilita que el trabajo efectuado sea verdaderamente colaborativo y por tanto, se llegue a las metas de aprendizaje propuestas.

- Comprender que el trabajo en equipo no es sencillo, pero constituye una experiencia relevante en la vida profesional. Los alumnos deberán tratar de resolver los conflictos de sus equipos por sí mismos y solicitar ayuda, únicamente en caso de requerirla.

- Mantener el contacto con el profesor a través de uno de los integrantes del equipo para expresarle sus dudas y problemas. La incertidumbre forma parte de esta estrategia de trabajo, el sentirse un tanto intranquilo por la nueva experiencia es natural.

- Administrar el tiempo adecuadamente.

- Mantener un proceso de análisis permanente de cada elemento nuevo que se vaya acumulando o incorporando producto de la investigación personal (por cada miembro del grupo) o como resultado de la discusión y el trabajo colaborativo.

Con respecto al aprendizaje-enseñanza de las matemáticas, se demanda, adicionalmente, definir conceptos como: matematizar, contexto, entorno, e incluso, problema, ellos revelan las dificultades que deberá enfrentar la implementación del nuevo programa de estudios. Resulta lamentable que tales aspectos no hayan sido profundizados en el marco teórico o conceptual de la propuesta, así como la exclusión, igual que ocurrió en el siglo XX, de las correspondientes apreciaciones en cuanto al impacto emocional generado en la educación matemática.

Desde la perspectiva de este ensayo, el éxito en la implementación de dicha propuesta requiere de una reconceptualización de muchos aspectos; sin embargo se primará la relación entre el lenguaje ordinario y el matemático, por considerar la dependencia del segundo con respecto al primero, aspecto que no es tomado en cuenta de manera deliberada y explícita 
en los documentos respectivos, en los cuales, además, no se detalla la forma en que deberán examinarse las paradojas propias de las matemáticas. Paradojas que versan sobre el doble papel de las matemáticas como instrumento-herramienta para la solución de problemas, y su condición de contenido propio de un área del conocimiento humano, así como las diferencias en la doble representación en que se puede presentar el lenguaje matemático, ya sea algebraica (semántica) o gráfico-visual (icónica), las cuales no son isomórficas.

Como consecuencia, se le presenta al lector una reflexión sobre el perfil del aprendizaje y la enseñanza de las matemáticas, la cual parte del nuevo programa de aprendizaje-enseñanza de las matemáticas para la educación media en Costa Rica, y centra su interés en la relación que existe entre el lenguaje ordinario y el matemático, y por qué el primero constituye el eje transversal para el aprendizaje de las matemáticas desde la perspectiva del ABP. Se pretende así, destacar la importancia del lenguaje ordinario como un instrumento de trabajo que contribuye a la modelización matemática del entorno y su relación con el contexto.

Asimismo, se sugiere que el manejo correcto del lenguaje ordinario se valore como la clave para que los educandos logren un aprendizaje más significativo de las matemáticas, mediante la solución de problemas, por su influencia en la generación de las habilidades, destrezas y competencias necesarias para que, en su proyección como futuros profesionales, puedan insertarse, con posibilidades de éxito, en el modelo socio-económico imperante.

\section{La necesidad de un nuevo programa de educación matemática en Costa Rica}

A finales de los años cincuenta se dio una reforma mundial en la enseñanza de las Matemáticas, a ella se sumó Costa Rica. De este cambio derivó que la enseñanza de las matemáticas se basara en la teoría de conjuntos, estructuras y sistemas axiomatizados, lo que conllevó a un manejo formalista, deductivo y abstracto (Ruiz y Chavarría, 2003). Esta forma de enseñar y pretender el aprendizaje de las matemáticas no logró elevar el nivel del pensamiento abstracto de los educandos sino que, por el contrario, evidenció serias limitaciones; por ejemplo, para finales del siglo XX la enseñanza y el aprendizaje se enfocó en su algebrización (Artigue, 1998), aspecto que, posteriormente, ocasionó el uso excesivo de las calculadoras, más allá de su calidad de instrumento (García, 2009a). Estos modos de 
enseñar y de aprender matemáticas se dieron en contra de su naturaleza histórica, la cual se ha caracterizado por basarse en una aproximación empírica, y por ende, pragmática, a los problemas que plantea, previa a su teorización (González y Waldegg, 1995).

En efecto, se puede afirmar que el pensamiento abstracto y la deducción, propios del pensamiento aristotélico y occidental, siempre han ido a la zaga de los problemas planteados por la naturaleza o las relaciones sociales, es decir, la aproximación empírica a los problemas con la ayuda de las matemáticas (dígase solución de problemas, modelización matemática, etc.) ha antecedido siempre a su abstracción (González y Waldegg,1995), por lo que el aporte de las matemáticas a múltiples disciplinas siempre se ha caracterizado por ser de carácter pragmático o reflexivo más que teórico. El nuevo programa deviene entonces en la respuesta necesaria para enfrentar la teorización, la algebrización y sobre todo el uso de las calculadoras como sustitutos del pensamiento matemático (García, 2009a), y evidencia el interés por dar un carácter histórico, coyuntural y contextual a los aprendizajes, dado que éstos se construyen en un tiempo y lugar específicos y responden a necesidades objetivas y concretas de la sociedad, se constituyen en razón de su uso y comunicación, más como formas de actividad que como resultado de la unión de distintas visiones culturales (Gómez, 1991). Adicionalmente en el mismo se asume que no existe una receta para la educación matemática y que el esfuerzo del aprendizaje debe recaer en el aprendiz, de manera tal que el papel del docente consista en ser un mediador entre el conocimiento, las habilidades, destrezas y las competencias a ser adquiridas o desarrolladas por los estudiantes.

Por consiguiente, el programa plantea, como reto a los docentes, la implementación de un nuevo currículo adaptable a la realidad de todos los participantes del acto educativo, así como llegar a comprender que de ellos depende el potenciar, inducir o dificultar el desarrollo de procesos metacognitivos capaces de "impulsar y consolidar en sus estudiantes hábitos, disposiciones y competencias para la regulación autónoma de las prácticas meta-cognitivas" (Rigo, Paéz, y Gómez, 2009, p. 444). El objetivo consiste en lograr que los educandos desarrollen competencias aptas para comprender sus condiciones materiales de vida y sean capaces de transformarlas como parte de una relación empática con su entorno.

El desarrollo de este nuevo enfoque del aprendizaje y enseñanza de las matemáticas se ejecuta en un contexto dinámico, en el cual el papel de la información actúa como base para la construcción del conocimiento, el cual, a su vez, se ha convertido en un recurso económico por excelencia. Esto significa que el objetivo macrosocial perseguido por el programa estriba en contribuir a que los estudiantes sean capaces de utilizar las 
matemáticas y el pensamiento matemático como un recurso más que posibilite potenciar el desarrollo económico. Es decir, el programa pretende que el conocimiento matemático logre articularse con la idea de que el conocimiento se ha convertido en la principal fuerza de producción (Lampert, 2008).

En la actualidad, el saber y el conocimiento se han reducido a información y procesamiento de esta, con el propósito de constituirse en el principal insumo para la producción, aspecto clave para lograr la competitividad en el campo económico y empresarial (Hopenhayn, 1987) y así, garantizar una inserción en la economía globalizada con posibilidades de éxito.

Lo anterior, particularmente, resulta importante en áreas profesionales como las ciencias económicas o las ingenierías, las cuales dependen de las matemáticas y son vitales para las economías modernas, áreas en las que se muestran serias debilidades así como un alto déficit de estudiantes universitarios en tales carreras, al punto de que, según la encuesta Escasez de Talento, realizada por Manpower Group en 2012 (Manpower Group, 2012), la falta de profesionales en estos dos campos ocupan respectivamente los lugares 1 y 2 dentro de una lista de los 10 profesionales más difíciles de cubrir.

\section{La importancia de definir el contexto para el ABP}

La metodología del Aprendizaje Basado en Problemas (ABP) no constituye una propuesta reciente, desde la década de los 80 del siglo XX ya había sido planteada, entre otros por Howard Barrows (Instituto Tecnológico de Monterrey, 2010). En el caso de Costa Rica, esta metodología no se había considerado, al menos en el campo del aprendizaje y enseñanza de las matemáticas, hasta la presentación de la propuesta actual con la finalidad de pasar de un modelo educativo basado en una acumulación enciclopédica, discursivamente constructivista, apoyado en el uso y abuso de las calculadoras científicas (García, 2009a), hacia un modelo más dinámico. De hecho para el MEP el ABP se plantea como parte de un conjunto de estrategias metodológicas que posibiliten los siguientes procesos:

Así por ejemplo, se plantea el uso de varias estrategias, que entre otras, incluyen cinco procesos básicos:

- Razonar y argumentar.

- Plantear y resolver problemas.

- Conectar, establecer relaciones. 
- Representar de diversas formas.

- Comunicar, expresar ideas matemáticas formal y verbalmente. (MEP, 2012, p. 11).

Esto evidencia los esfuerzos por lograr un ajuste del modelo educativo costarricense a los lineamientos que, al respecto, han surgido desde la Organización para la Cooperación y el Desarrollo Económico (OCDE, 2010) con el propósito de que un país se pueda insertar en dicha organización como parte de la economía globalizada. Consecuentemente, dicha propuesta constituye un elemento estratégico donde se destaca la importancia y necesidad de ligar la interacción entre el educando, sus situaciones de aprendizaje y los contextos sociales, económicos y culturales en los cuales participa (Gómez, 1991), a fin de poder responder a la globalización de la economía.

En el caso de la educación matemática, una de las grandes virtudes y posibilidades que ofrece el uso del ABP está en la capacidad de modelización a partir del manejo de problemas. En el programa se entiende por modelización matemática del entorno a la "identificación, manipulación, diseño y construcción de modelos matemáticos sobre situaciones auténticas del entorno" (MEP, 2012, p. 32). Esta consideración presenta un cierto carácter tautológico, aunque procura evitar que se generen contradicciones entre competencias y aprendizajes matemáticos específicos para "entornos específicos". Igualmente, destaca la importancia de un aprendizaje holístico y heurístico que potencie la capacidad de apreciar, en las matemáticas, un instrumento que contribuya a la solución de problemas que se dan en el entorno de los sujetos (García, 2013).

La modelización trae consigo el problema de definir qué debe ser entendido por "entorno". Según el diccionario de la Real Academia Española de la Lengua la palabra entorno significa "ambiente, lo que rodea" (Real Academia Española de la Lengua, RAE, 2001), lo cual se debe ligar con el concepto de "contexto" por ser más genérico. Este, según la RAE (2001) se entiende como "Entorno físico o de situación, ya sea político, histórico, cultural o de cualquier otra índole, en el cual se considera un hecho", o bien como "Entorno lingüístico del cual depende el sentido y valor de una palabra, frase o fragmento considerados" (RAE, 2001). Por lo anterior, entorno y contexto abarcan componentes objetivos, subjetivos e interpretativos en cada individuo, lo cual demanda esfuerzos de consenso y entendimiento para ser considerados socialmente.

Esta situación se torna aún más complicada si se considera que los problemas a ser tratados, como parte del ABP, demandan ser "leídos", interpretados y entendidos como 
"situación en contexto", aspecto que depende de los individuos, por lo que no necesariamente significan lo mismo para los educandos y los educadores. A lo anterior debe agregarse que los contextos entre una región y otra pueden presentar diferencias exacerbadas en muchos aspectos. Muestra de ello, en el caso de Costa Rica, el Índice de Desarrollo Social (IDS), elaborado por el Instituto de Fomento y Asesoría Municipal (IFAM, 2003), varió desde un 0 en el cantón de Talamanca hasta un 100 en el cantón de Flores a lo que habría que añadir, con base en el 19 Informe del Programa Estado de la Nación de 2013 que: "La evolución del cociente Gini recalculado desde 1987 muestra que el país está entrando en una nueva etapa de elevada desigualdad" (Programa Estado de la Nación, 2013, pág. 99) y: "El ingreso total promedio del $10 \%$ de los hogares más ricos (décimo decil) es 24.8 veces mayor que el $10 \%$ más pobre (primer decil)" (p. 100).

Otros elementos que contribuyen a denotar la existencia de contextos desiguales están constituidos por las brechas en el acceso a las tecnologías digitales, así como a la internet, lo que hace que mientras unos educandos pueden interactuar y aprender tanto en entornos virtuales como naturales, otros están limitados a tan solo el segundo, o de manera parcial, incluyen al primero. Lo anterior obliga el aceptar la existencia de distintas realidades y contextos de modo tal que los problemas planteados como parte de la estrategia del ABP serían diferentes según la ubicación geográfica, social, económica, cultural y tecnológica de los participantes, aspectos que inciden en la percepción de qué significa un problema y cómo solucionarlo. Es decir, el ABP, tal y como se ha planteado en la propuesta ignora que el modelo educativo costarricense no es homogéneo entre las comunidades de bajos recursos económicos o lejanas a los grandes centros poblacionales y las comunidades que cuentan con recursos abundantes (García, 2014).

A esto debe agregarse que, dependiendo de la experiencia, las características personales o los conocimientos acumulados, tanto por los educadores como por educandos, la solución de problemas puede considerarse desde una perspectiva secuencial y/o algorítmica, ligada fuertemente al lenguaje y contempla hacer planes, organizar el tiempo, presupuestar, optimizar recursos y finanzas, realizar un abordaje con carácter técnico, ecológico, social, para llegar a una toma de decisiones; o bien, puede ser considerado desde una perspectiva mecánica y superficial que, en términos generales, se caracterice por apoyarse en el uso de tecnologías digitales y particularmente calculadoras (García, 2009a).

Lo anterior, sugiere la importancia de definir un marco de trabajo que considere el negociar entre todos los participantes del acto educativo. Asimismo, interrogantes del tipo: 
¿Qué se deberá entender como situación y contexto?, ¿qué es un problema bajo esas condiciones?, ¿qué papel debe jugar el lenguaje? y ¿qué impacto pueden tener las tecnologías digitales? Sería riesgoso ignorar la discrepancia que con respecto a estos aspectos puedan mostrar educandos y educadores. No obstante, es importante destacar que entre las grandes virtudes del ABP que se pretenden implementar, está su capacidad para homogenizar el proceso de aprendizaje-enseñanza de las matemáticas desde una perspectiva metodológica común a todos los contextos.

\section{El concepto "problema" para el aprendizaje de las matemáticas}

El potencial conflicto sobre qué son y cómo se interpretan los contextos incide directamente en lo que cada uno de los participantes del acto educativo puede considerar como "problema", esto hace necesario consensuar que será considerado como tal. El lenguaje ordinario cobra un papel relevante para determinar qué se deberá entender por "problema", y particularmente por problema matemático. El nuevo programa de educación matemática en Costa Rica indica al respecto:

Un problema es un planteamiento o una tarea que busca generar la interrogación y la acción estudiantil utilizando conceptos o métodos matemáticos, implicando al menos tres cosas:

1) que se piense sobre ideas matemáticas sin que ellas tengan que haber sido detalladas con anterioridad,

2) que se enfrenten a los problemas sin que hayan mostrado soluciones similares,

3) que los conceptos o procedimientos matemáticos a enseñar estén íntimamente asociados a ese contexto (MEP, Programas de Matemáticas 2012, p. 29).

Esta definición demanda que el educando sea capaz de manipular tanto el lenguaje ordinario como el lenguaje matemático, e implícitamente, la capacidad para trasladarse de un lenguaje a otro. Por su parte, la definición resulta tautológica y no aclara lo que realmente debe ser entendido como un problema desde el aprendizaje de las matemáticas, ni cómo este contribuirá con el desarrollo de competencias merced al manejo del ABP. Esto no resulta sorprendente, para Parra (1990) se dificulta lograr un consenso acerca de lo que se puede entender como problema en matemáticas debido a que distintos autores apuntan a aspectos que consideran particularmente importantes desde su óptica. Como consecuencia, 
los conceptos de "problema" y "solución de problemas" han tenido y tienen múltiples significados contradictorios.

En razón de lo anterior, en el presente ensayo, sin ánimo de polemizar con el nuevo programa de educación matemática, se considera que la propuesta de Schoenfeld (1992) está implícitamente inserta en el nuevo plan. Schoenfeld partió de considerar el método propuesto por George Polya compuesto por los siguientes pasos: en primer lugar, entender el problema; en segundo lugar, configurar un plan; en tercero, ejecutar el plan y por último, comprobar el resultado. El autor concluyó que la misma demanda que el aprendiz sea capaz de comprender la situación que debe enfrentar en la medida que la respuesta al problema no aparezca de manera inmediata.

Para Schoenfeld la propuesta de Polya resulta ser muy esquemática y no toma en cuenta cuatro factores que él considera fundamentales: a) las heurísticas (estrategias cognitivas), b) los sistemas de creencias (tanto del educando como del docente), c) los recursos (conocimientos previos o el dominio de ellos) y d) el control o estrategias metacognitivas (Chavarría y Alfaro, 2005). Tales aspectos evidencian la complejidad que conlleva el resolver problemas, lo que demanda de los aprendices ejecutar distintas actividades, garantizando un control ejecutivo en calidad de "regulación consciente y deliberada de su conducta, de manera que para realizarlas se vean obligados a planificar previamente su actuación" (Moreno, 2012, p. 5).

A esto habría que agregar que resolver problemas demanda la capacidad de transformación del lenguaje ordinario, a través del cual se presentan al lenguaje matemático, instrumento con el que se trabaja, ya sea en términos algebraicos (semánticos) o gráficovisuales (icónicos), en calidad de recursos de apoyo para la comprensión y traslación de los conceptos e información implicados en el problema. Aunado a esto se encuentra la capacidad del educando para que, una vez resuelto el problema, pueda regresar al lenguaje ordinario a fin de poder exponer sus resultados. Sin embargo las transformaciones de un lenguaje a otro no siempre se toman en cuenta de manera explícita al momento de enseñar matemáticas, situación que propicia una pérdida de coherencia con respecto al tipo de instrumentos que se requieren utilizar a la hora de resolver problemas.

Se refleja así que el aprendizaje de las matemáticas conlleva una gran complejidad cognitiva, máxime que las representaciones algebraicas (semánticas) o gráfico-visuales, a través de las cuales se trata de manipularlos, muchas veces son muy diferentes entre sí e 
implican el uso de recursos/instrumentos cognitivos distintos y dispares, tanto por parte de los docentes como de los estudiantes.

A modo de recapitulación, en este ensayo se considera como problema a una situación, planteamiento o tarea propuesta desde el lenguaje ordinario, el cual involucra una interrogante a responder, para así, a través de su recodificación en lenguaje matemático, ya sea en forma algebraica, gráfico-visual, o de una combinación de ambas se procure la manipulación de la información y conceptos con que se cuenta de una manera más clara y simple posible, de modo que contribuya a responder a la interrogante planteada.

\section{El lenguaje ordinario es la base para generar el lenguaje matemático}

El lenguaje ordinario es el resultado de la actividad humana, posee una dimensión histórico-económica y depende de una superestructura simbólica (Radford, 2004), igualmente constituye una herramienta mental (cognitiva) muy compleja de definir. En el presente trabajo se le considera como una herramienta primaria de carácter socio-cultural facilitadora de la comunicación y con ello de la adquisición de otras herramientas. También, se utiliza en muchas funciones mentales como la atención, la memoria y el pensamiento lógico-matemático (Vigotsky, 2005), Finalmente, se caracteriza por poseer un carácter contextual, por generar nuevos contextos, e involucrar aspectos cognitivos, racionales, afectivos y emocionales en su intención de construir significados.

Todo lenguaje posee tres aspectos claves, el primero un conjunto de elementos básicos discretos o vocabulario; el segundo, una sintaxis, es decir, un determinado poder para combinar los elementos del vocabulario de manera específica, donde cada secuencia puede determinar conceptos diferentes; y por último un alcance semántico personal, generado por secuencias sintácticamente bien formadas, que permiten al individuo hablar sobre objetos (Quesada, 1991). Esto significa que el lenguaje es como un texto que se produce y se revisa constantemente, cambia en razón de la acumulación de información, reflexiones posteriores o por la influencia de las emociones y los sentimientos. Las matemáticas vistas como lenguaje no escapan a este proceso ya que los objetos de los que habla y con los que trata se encuentran en permanente construcción, siendo esta la parte más problemática y por ende más rica de su significado (D`Amore, 2011).

El lenguaje ordinario es el tipo más complejo de comunicación intencional (Ramos, s.f.) que relaciona sistemáticamente símbolos con significados y establece reglas para combinar y recombinar tales símbolos con la finalidad de ofrecer diferentes tipos de información. 
Constituye un sistema arbitrario de signos abstractos reconocido por un grupo de personas del que se sirven para comunicar sus pensamientos y sentimientos, en él, el signo cumple una función mediadora entre el individuo y el contexto, con lo cual permite pasar de lo intrapsicológico a lo interpsicológico (Vigotsky, 2005). Al considerar al lenguaje ordinario como punto de partida para la construcción del lenguaje matemático, se evidencia la existencia de una compleja relación entre signo y significado, aspecto que se agrava por un paradójico uso de símbolos que no poseen referentes físicos y demandan un manejo totalmente conceptual. Se cree que a partir de ellos, el individuo debe ser capaz de modelar la realidad, a pesar del carácter polisémico que, muchas veces, tales símbolos engloban.

El lenguaje ordinario contribuye a la interpretación que cada persona o grupo humano hace del entorno, lo que hace inevitable que posea un carácter polisémico. Su incremento conlleva un enriquecimiento del campo semántico del individuo, lo cual potencia su capacidad de contextualización. Al ser estos aspectos trasladados al lenguaje matemático, se refleja la competencia comunicativa de las matemáticas como una de las cuestiones más importantes y significativas (Pimm, 2002), por cuanto "lo que conocemos y la manera en que llegamos a conocerlo está circunscrito por posiciones ontológicas y procesos de producción de significados que dan forma a cierta clase de racionalidad que permite plantear ciertos problemas" (Radford, 2004, pág. 13).

\section{La diferencia entre los lenguajes ordinario y matemático, elemento clave para comprender la dificultad del aprendizaje de las matemáticas}

El aprendizaje de las matemáticas parte del lenguaje ordinario para la construcción de los conceptos básicos o fundamentales que utiliza, aunque conforme se desarrolla, genera y utiliza su propia simbología, sintaxis y semántica para manipularlos. Consecuentemente, el aprendizaje de las matemáticas se torna paradójico, ya que puede constituir un instrumento de trabajo para resolver problemas o un contenido de aprendizaje en sí mismo. Muchas disciplinas recurren a las matemáticas y su lenguaje en procura de lograr la solución de problemas que les competen, y en este sentido constituyen herramientas o instrumentos de trabajo. No obstante, las matemáticas también poseen contenidos de conocimientos por si mismas que incluyen problemas propios demandantes de un manejo específico.

En el aprendizaje de las matemáticas conviene tomar en cuenta la paradoja de Duval (2006), la cual establece que para que el educando pueda aprender matemáticas requiere desarrollar la capacidad de "representación", la cual en términos generales, como se ha 
indicado, puede ser de dos tipos: la algebraica (asociada usualmente a la semántica) y la gráfico-visual (asociada con dibujos o íconos). Mediante estos dos recursos, el educando debe procurar interpretar la "situación-problema" para determinar que procedimiento es el más apropiado (eficiente y eficaz) a seguir con el fin de solucionarlo, pero el que este conozca o aprenda ( $\mathrm{y}$ aprehenda) tales recursos, no garantiza que pueda llegar a interpretar correctamente los problemas y mucho menos resolverlos.

Es decir, para solucionar problemas se requieren instrumentos, pero su posesión no garantiza el poder resolverlos. Ser capaz de interpretar los problemas no implica el poseer los instrumentos necesarios para solucionarlos. La capacidad para interpretar una situaciónproblema y poder trasladarla del lenguaje ordinario al lenguaje matemático, demanda contar con las herramientas matemáticas necesarias, aunque el poseer tales mecanismos no conlleva automáticamente la capacidad de poder resolver problemas.

En este singular marco, el docente juega un papel trascendental, porque, independientemente del enfoque epistemológico y didáctico que asuma, su percepción del qué y para qué de las matemáticas será trasladada a los educandos. Así, el aprendizaje de las matemáticas puede ser visto por el docente como un "para qué" (en calidad de instrumentos), o un "por que" (en calidad de contenido) que posee sus propios problemas, aspecto que afecta la manera cómo realiza la enseñanza, es decir, el enfoque al que recurrirá ya sea por medio de un manejo algebraico (semántico) o gráfico-visual.

En efecto, existen evidencias de que las prácticas de aprendizaje están determinadas por el enfoque que sigue el docente con respecto a cómo percibe, manipula y transmite los conceptos matemáticos (Bayazit, 2010). Como consecuencia, tal aprendizaje difiere, en términos cualitativos, de unos a otros estudiantes, de un docente a otro, de un contexto a otro, de una carrera a otra, e incluso, de un curso a otro.

De ahí que el papel que desempeña el docente es clave para no caer en un círculo vicioso analizado por Duval (1993 citado por D’Amore, 2011), para quien el aprendizaje de los objetos matemáticos debe ser conceptual, por causa de la naturaleza de tales objetos. Esto es posible, únicamente, si se realiza a través del propio lenguaje matemático, el cual permite el manejo conceptual de tales objetos, es decir, solo se puede aprender a manipular los objetos matemáticos si se cuenta con el lenguaje matemático, pero para contar con el lenguaje matemático se requiere poder manipular los objetos matemáticos. Ante ello, Duval se plantea los siguientes cuestionamientos: 
¿Cómo sujetos en fase de aprendizaje no podrían confundir los objetos matemáticos con sus representaciones semióticas si ellos no pueden más que tener relación solo con dichas representaciones? (...) Y al contrario ¿cómo podrían ellos adquirir el dominio de los tratamientos matemáticos, necesariamente ligados a las representaciones semióticas, si no tienen ya un aprendizaje conceptual de los objetos representados? (Duval 1993, citada por D’Amore, 2011, p. 6)

Algunos ejemplos ilustrativos de esta situación conflictiva son los enunciados a continuación:

a) El símbolo “-“, este posee una gran cantidad de acepciones. El primer acercamiento ocurre en la educación primaria para identificar el concepto de "resta". De este modo los párvulos lo asocian con la idea de que a un todo se le separa una parte que es menor al todo, por lo que, de manera natural, el resultado es un número positivo. Dicha aproximación conlleva una correspondencia muy directa entre los lenguajes ordinario y matemático.

Aún así, al llegar a la educación media, el símbolo comprende múltiples conceptos. La resta deja de estar abiertamente presente y aparece como un recurso para representar números negativos, "Para el pensamiento occidental, sustentado por el principio de contradicción e igualdad, el número negativo no es algo natural" (Radford, 2004, pág. 12). Tales números tienden a convertirse en un serio problema cuando se asumen los criterios utilizados durante el renacimiento occidental para identificar, a través de ellos, a las "deudas" (Radford, 2004). Como contraparte se tiene el "+" para las ganancias, porque llevan al insólito caso de que al multiplicar dos números negativos, una deuda por otra, implicaría generar ganancias.

Adicionalmente, si el aprendizaje de los números negativos se enmarcara desde una perspectiva de "direcciones", el lenguaje ordinario contribuiría a considerar que tal signo implicaría, en el caso de una recta numérica, estar a la izquierda de cero. Esta identificación con una dirección permitiría hablar de "direcciones opuestas", y por ende, del opuesto de un número, donde la expresión “- - b”, expresaría la dirección opuesta de un número negativo, lo que hace que el "-“" esté refiriéndose a un número positivo. Es decir, el "-“" no siempre representa a un número negativo. Para concluir, el uso del "-“ debe ser considerado contextualmente. Este carácter contextual alcanza su máxima expresión al considerar expresiones como $\lim _{x \rightarrow-b} f(x)$ donde el símbolo “_“ indica que las pre-imágenes de una 
función se acercan por la izquierda de un número negativo (asumiendo que b es positivo), lo cual para estudiantes novatos, en recurrentes ocasiones, resulta confuso (Engler, Gregorini, Vrancken, Müller, Hecklein, y Henzenn, 2008).

b) Un símbolo aparentemente tan simple como "0", y su capacidad para representar la "ausencia" resulta sorprendente cuando se debe usar para completar un polinomio, ya que lejos de representar la ausencia de algo, el "no hay", constituye el recurso fundamental para indicar la existencia de otras "cosas" que permiten hablar de orden, crecimiento y completitud.

c) Directamente ligado a la relación entre los lenguajes ordinario y matemático, el traslado de uno al otro puede resultar confuso, aspecto que incide significativamente al momento de querer resolver problemas. Por ejemplo, solicitar a los estudiantes trasladar del lenguaje ordinario al algebraico, la expresión "el doble de un número aumentado en tres" (*). Experimentos realizados por este autor muestran que muchos estudiantes tienden a escribir " $2 x+3$ ", pero enfrentan un choque cognitivo si se les plantea como alternativa " $2(x+3)$ ". Tal choque se incrementa cuando después de ser escrito el "texto" en la pizarra, éste se lee muy rápidamente y se compara con las expresiones algebraicas.

Los estudiantes novatos, generalmente, no logran dar con las diferencias entre ellas, no es, sino posterior a realizar varias lecturas, cada vez más lentas, marcando las correspondientes entonaciones: "el doble de un número... aumentado en tres", y diferenciándola de: "el doble de... un número aumentado en tres", que demandan en el lenguaje ordinario escrito denotarlas con comas, que se evidencia a la expresión inicial $\left(^{*}\right)$ como técnicamente intransferible del lenguaje ordinario al lenguaje matemático.

Los ejemplos anteriores muestran varias cosas a ser tomadas en cuenta cuando se pretende resolver problemas, primero la dificultad, y en algunos casos la imposibilidad, de homologar el lenguaje ordinario con el matemático; la segunda, el que el lenguaje matemático combina simbología con contenido; tercera, que el lenguaje matemático es polisémico y contextual, depende de lo que se hable, como se hable y que se quiera representar. Consecuentemente con ello, las constantes repeticiones por parte del docente, si no muestran enfoques diversos, no contribuyen a que los estudiantes logren "asir" los conceptos, comprender el problema o poder determinar como trasladarse de un lenguaje a 
otro, lo cual contribuye a que el aprendizaje de las matemáticas termine dentro de un aire de misterio que es apabullado por la algebrización en su manejo (Artigue, 1998, Tall, 1992).

Los ejemplos permiten considerar que el deficiente aprendizaje de las matemáticas, por parte de muchos estudiantes, podría residir en la dificultad que enfrentan para entender aquello de lo que se les habla o se les cuenta, o bien en la forma cómo se les cuenta y el para qué se les cuenta (Ortega y Ortega, s.f.), por lo que el no lograr pasar del lenguaje ordinario al lenguaje matemático, debido al carácter polisémico y contextual que ambos presentan, puede conllevar a que la traslación-traducción genere vacíos conceptuales y procedimentales imposibles de cubrir.

\section{El lenguaje ordinario como eje transversal para resolver problemas en matemáticas}

En 2009, en el ensayo "El aprendizaje de las matemáticas por medio de la solución de problemas" (García, 2009b) se presenta una propuesta pedagógica para el aprendizaje de las matemáticas por medio de la solución de problemas. Esta proposición fue planteada varios años antes que la del MEP, resulta ajena a ella, consta de siete pasos: leer, comprender, traducir, construir mecanismos alternativos para la búsqueda de la solución, aplicar algoritmos específicos u operaciones, determinar posibles respuestas incluyendo la selección y comprobación, y brindar resultados o respuestas.

La propuesta fue elaborada desde una perspectiva metodológica y con un sentido metacognitivo, con la finalidad de ofrecer una alternativa para que los docentes y educandos pudiesen tomar conciencia del proceso cognitivo que ponen en marcha cuando procuran solucionar problemas utilizando matemáticas. El autor considera que los pasos propuestos no se ejecutan en estado "puro", es decir, no aparecen diferenciados unos de otros de forma clara y distinta, sino que evidencian la existencia de muchos enlaces entre ellos, lo que hace que su "identificación y aislamiento" deba ser sopesado de manera relativa y por el papel cognitivo que cada uno desempeña a partir de considerar que los aprendizajes que se van construyendo son reforzados y consolidados por la reflexión del educando sobre los mismos (Lacon y Ortega, 2008).

Las etapas leer, comprender y traducir implican un manejo del lenguaje ordinario que, gradualmente, debe ser trasladado al lenguaje matemático. En otras palabras, constituyen una zona de interacción entre los dos lenguajes, y evidencian el nivel del desarrollo cognitivo del educando, así como la capacidad para ir más allá de lo superficial ya que demandan una 
organización de la información que posibilite una actividad tanto cognitiva como metacognitiva, merced a la formulación y reformulación del texto. Tales etapas se pueden ubicar dentro de los que Moreno (2012) denomina conocimiento declarativo, por lo que su manejo correcto debe ir más allá del uso mecánico e instrumental de la simbología.

La epata "leer" se plantea más allá de las competencias propias de la lecto-escritura porque incluye una "lectura del entorno", en la cual es imprescindible conocer y comprender el carácter semántico de los términos utilizados, la manera como se enuncie (se proponga) un problema (lo cual implica más que fonetizar las palabras) es determinante para establecer una relación entre los términos utilizados, el contexto y el acervo histórico y cultural de los participantes. Estos representan aspectos que deben tener sentido y significado tanto individual como social, deben ubicarse dentro de un marco contextualmente consensuado, ya que existe la posibilidad de que la lectura realizada por el docente no coincida con la efectuada por el educando.

No debe sorprender que para muchos docentes el concepto de lectura remite a textos "físicos", mientras que para muchos estudiantes implica textos "virtuales", por lo que debe ser considerada también desde estas dos dimensiones. En síntesis, la lectura debe estar ligada al contexto y la realidad, demanda interpretar las cosas que existen o suceden en el entorno de manera consensuada, y sería ideal que esté orientada a estimular la motivación, acaparar la atención y proponer retos posibles al educando.

En la etapa "comprender", el aprendiz debe utilizar el lenguaje ordinario con el objetivo de perfilar la intención del problema, es decir, establecer qué es lo que se pretende solucionar, qué es lo que el problema quiere que realice. Comprender requiere contextualizar, en términos tanto del entorno general, como del marco específico al que se restringe el enunciado. Esta etapa es crítica cuando la historia del aprendizaje de la lectoescritura del educando ha mostrado debilidades, o cuando el vocabulario y el campo semántico son pequeños.

Asimismo, la compresión implica realizar un análisis que permita separar la información útil de aquella que es superflua, lo que se traduce en un proceso de clasificación de la información según su importancia o relevancia. Las emociones cobran una fuerza extraordinaria y exigen al aprendiz enfrentarse a su pasado y poner en juego el acumulado de sus experiencias relativas al tema que se trabaja. En el caso del docente, comprender el problema incluye dimensionar la dificultad que posee para que no exceda el nivel y capacidad de los educandos y se convierta en un elemento frustrante. 
En lo que corresponde a "traducir", esta etapa junto a la anterior, constituyen las fases en las que los estudiantes enfrentan muchas dificultades. La traducción consiste en la traslación del lenguaje ordinario a un conjunto de símbolos propios del lenguaje matemático, sean estos algebraicos o gráfico visuales. Demanda un análisis de la información para determinar cuáles referentes matemáticos son los más apropiados con los cuales puede asociar la situación planteada con la que ya conoce, de esta manera puede separar lo relevante de lo irrelevante, así como diferenciar los elementos explícitos de los implícitos. Cuando se logra lo anterior, el educando puede pasar del conocimiento declarativo al procedimental y establecer las condiciones que permitan trabajar con una serie de símbolos manipulables de manera eficiente, como si fueran parte de una pequeña maquinita (Radford, 2004).

Las etapas de leer, comprender y traducir constituyen constituye la parte medular del proceso de matematización del problema. De manera conjunta se pueden considerar como un proceso de traslación-transferencia que depende del nivel de desarrollo del "lenguaje interior" del aprendiz. El lenguaje interior actúa como elemento intermedio entre los lenguajes ordinario y matemático, que son lenguajes sociales, y constituye un instrumento intelectual capaz de ligar los elementos previamente conocidos o desarrollados por el aprendiz, guardados en la memoria a largo plazo que pueden ser útiles ante la situación con que se enfrenta. Es a través del lenguaje interior que el individuo puede reconocer, identificar y homologar situaciones resueltas previamente; lo que permite establecer la complejidad que el problema conlleva, poner en juego la creatividad y experiencia.

\section{Conclusiones}

El nuevo programa de estudios en el área de las matemáticas asumido por el MEP desde 2012 para la Educación General Básica y el Ciclo Diversificado, ofrece una nueva alternativa a los estudiantes para que puedan empoderarse, como nunca antes se había hecho, con respecto a su aprendizaje. Dicha herramienta establece la necesidad de reconocer el entorno y la presencia de las matemáticas en él, una vez logrado esto pueden recurrir al lenguaje matemático como un mecanismo de comunicación que surge del lenguaje ordinario.

Sin embargo, conceptos como entorno, contexto, problemas y lenguaje, son difíciles de definir o precisar porque todos dependen de consideraciones tanto objetivas como subjetivas. Estos elementos, trasladados al modelo educativo en propuestas que pretenden 
ser "concretas", requerirán siempre ser consensuados si se pretende con ello lograr impactos positivos. Las múltiples interpretaciones de las que pueden ser objeto demandan un diálogo permanente a nivel de la superestructura institucional como a nivel de aula.

El aprendizaje de las matemáticas puede ser considerado desde dos ángulos: como instrumento de trabajo para resolver problemas, o como contenido de conocimiento en si mismo. Tal aprendizaje depende del lenguaje ordinario, el cual a su vez es el resultado de la interacción social en la que participa el individuo. La calidad en el manejo del lenguaje ordinario y el enriquecimiento de su campo semántico, el cual puede ser estimulado desde la educación a través de la lecto-escritura, contribuyen a que la capacidad para aprender a resolver problemas pueda lograrse de una mejor manera.

El crecimiento del lenguaje ordinario y el consecuente enriquecimiento de su campo semántico actúan como facilitadores para la comprensión y uso hablado de los objetos, ya sean propios del lenguaje ordinario o del matemático. Estos aspectos podrían generar condiciones de reconocibilidad e identificación del objeto hablado distinguiendo sus posibles representaciones, lo que contribuye a que el lenguaje matemático crezca en cantidad y calidad, y por ende, a que su aprendizaje y utilización en la solución de problemas sea mucho más eficiente.

El hecho de partir de problemas del contexto proporciona una metodología que permite la homogenización en el tratamiento, a la vez que respeta la individualidad, este es posiblemente el mayor de los méritos del ABP. Sin embargo, tal metodología demanda de los docentes una consciencia crítica, un gran sentido de la realidad junto a la imaginación para evitar la mecanización de este extraordinario instrumento.

Todo lo anterior posibilita plantear que, probablemente, muchos de los posibles errores que cometen los estudiantes en el manejo del lenguaje ordinario son generadores potenciales de errores en la construcción, interpretación y valoración del significado de los objetos propios del lenguaje matemático, lo que podría llevar a hipotetizar que muchos de los problema del aprendizaje de las matemáticas estarían relacionados con un manejo débil o incorrecto del lenguaje ordinario.

En suma, se puede afirmar que el aprendizaje de las matemáticas está relacionado, e incluso, que depende en gran medida del aprendizaje del lenguaje ordinario, a tal punto que sus sintaxis y semánticas actúan de maneras similares. Comprender lo anterior, se vuelve vital para privilegiar el manejo de un lenguaje preciso y claro que contribuya, metacognitivamente, a adquirir consciencia sobre cómo se dan los procesos de aprendizaje 
para tratar de evitar aprendizajes mecánicos y memorísticos basados en la obtención de respuestas independientemente de su comprensión.

Las matemáticas, en tanto construcción humana, han sido creadas principalmente para resolver problemas de la naturaleza y de la vida cotidiana, lo cual no demerita el trabajo que realizan los matemáticos profesionales, mas este trabajo debe ser correctamente ubicado, por lo que es un error considerar que todo aquello que corresponde a la especialidad (particularmente la estructura y método), es susceptible de ser enseñado a toda la sociedad. De ahí, la necesidad de que los profesores de matemáticas tengan muy claro cuál es el contexto en que desarrollan su labor y cuáles son las dimensiones de su lenguaje ordinario y el de sus estudiantes para establecer la ruta a seguir.

\section{Referencias}

Artigue, Michèle. (1998). Enseñanza y aprendizaje del análisis elemental: ¿Qué se puede aprender de las investigaciones didácticas y los cambios curriculares? Revista Latinoamericana de Investigación en Matemática Educativa, 1(1). Recuperado de www.clame.org.mx/relime.htm

Bayazit, Ibrahim. (2010). The Influence of Teaching on Student Learning: The Notion of Piecewise Function. International Electronic Journal of Mathematics Education, 5(3), 146-164. Recuperado de www.iejme.com/

Chavarría, Jesennia y Alfaro, Cristian. (2005). Resolución de problemas según Polya y Schoenfeld. Ponencia presentada ante el IV Congreso Internacional sobre Enseñanza de la Matemática Asistida por Computadora (CIEMAC). Instituto Tecnológico de Cartago, Costa Rica. Recuperado de http://www.cidse.itcr.ac.cr/ciemac/memorias/4toCIEMAC/Ponencias/Resoluciondepro blemas.pdf

D’Amore, Bruno. (2011). Conceptualización, registros de representaciones semióticas y noética: interacciones constructivistas en el aprendizaje de los conceptos matemáticos e hipótesis sobre algunos factores que inhiben la devolución. Revista Científica. (11). Recuperado de http://www.dm.unibo.it/rsddm/it/articoli/damore/740\%20Conceptualizacion.pdf

Delors, Jacques. (1996). La educación encierra un tesoro. Informe a la UNESCO de la Comisión Internacional sobre la educación para el siglo XXI presidida por Jacques Delors. Madrid. Santillana, Ediciones UNESCO. Recuperado el 01 de Marzo de 2014 de http://www.unesco.org/education/pdf/DELORS S.PDF

Duval, Raymond. (2006). A cognitive analysis of problems of comprehension in a learning of mathematics. Educational Studies in Mathematics, 6, 103-131. Recuperado de www.cimm.ucr.ac.cr/ojs/index.php/eudoxus/article/download/162/297 
Engler, Adriana, Gregorini, María Inés, Vrancken, Silvia, Müller, Daniela, Hecklein, Marcela y Henzenn, Natalia. (2008). El límite infinito: una situación didáctica. Revista PREMISA. Sociedad Argentina de Educación Matemática (SOAREM). Año 10, № 36, págs. 1121. Recuperado el 31 de Enero de 2011 de http://www.soarem.org.ar/Documentos/36\%20Engler.pdf

García, José. (2009a). La Calculadora científica y la obtención de la respuesta correcta en el ciclo diversificado. Revista Actualidades Investigativas en Educación, 9(2). Recuperado de http://revista.inie.ucr.ac.cr/uploads/tx magazine/calculadora.pdf

García, José. (2009b). El aprendizaje de las matemáticas por medio de la solución de problemas. Revista Uniciencia, 23, Universidad Nacional, Heredia, Costa Rica.

García, José. (2013). La problemática de la enseñanza y el aprendizaje del cálculo para ingeniería. Revista Educación, 37(1). Recuperado de http://www.revistas.ucr.ac.cr/index.php/educacion/article/view/10627

García, José. (2014). Educación, Sociedad del Conocimiento y Tecnologías Digitales, de lo conflictivo a lo complementario. Ponencia presentada ante el V Taller Internacional "La Virtualización en la Educación Superior". $9^{\circ}$ Congreso Internacional de Educación Superior. La Habana, Cuba.

Gómez, Carmen. (1991) Cognición, contexto y enseñanza de las matemáticas. Comunicación, lenguaje y educación, (11-12). Recuperado de http://dialnet.unirioja.es/servlet/articulo?codigo $=126226$

González, María y Waldegg Guillermina. (1995). Lectura 3: El fracaso de la matemática moderna. La Matemática, su enseñanza y aprendizaje. Thais Castillo y Virginia Espeleta (Comps). San José, Costa Rica: EUNED.

Hopenhayn, Martín. (2002) El reto de las identidades y la multiculturalidad. Pensar Iberoamérica. Revista de la Organización de los Estados Iberoamericanos para la Educación, la Ciencia y la Cultura. Recuperado de www.oei.es/pensariberoamerica/ric00a01.htm

Instituto de Fomento y Asesoría Municipal. (2003). Regiones y cantones de Costa Rica. Dirección de Gestión Municipal. Sección de Investigación y Desarrollo. Recuperado de http://www.ifam.go.cr/docs/regiones-cantones.pdf

Instituto Tecnológico de Monterrey. (2010). Investigación e Innovación Educativa. Centro Virtual de Técnicas Didácticas. Recuperado de http://sitios.itesm.mx/va/dide2/tecnicas didacticas/abp/personajes3.htm

Lacon, Nelsi y Ortega, Susana. (2008). Cognición, metacognición y escritura. Revista signos, 41(67), 231-255. Recuperado de http://www.scielo.cl/scielo.php?pid=S071809342008000200009\&script $=$ sci arttext

Lampert, E. (2008). Postmodernidad y universidad: ¿Una reflexión necesaria?. Perfiles educativos, 30(120), 79-83. Universidad Nacional Autónoma de México. Recuperado de www.redalyc.org/articulo.oa?id=13211159005 
Manpower Group. (2012). Encuesta sobre escasez de talento. Recuperado de http://www.manpowergroup.com.mx/uploads/estudios/talentshortage d2012.pdf

Ministerio de Educación Pública (MEP). (2012). Reforma Curricular en Ética, Estética y Ciudadanía. Programa de Estudios de Matemática. I y II ciclo de la Educación Primaria, III Ciclo de la Educación General Básica y Ciclo Diversificado. San José, Costa Rica: MEP.

Moreno, María Guadalupe. (2012). La enseñanza de la resolución de problemas matemáticos. El banco y negro de algunas estrategias didácticas. Recuperado de http://www.quadernsdigitals.net/datos web/hemeroteca/r 24/nr 286/a 3699/3699.h $\underline{\mathrm{tm}}$

Organización para la Cooperación y el Desarrollo Económico (OCDE). (2010). Habilidades y competencias del siglo XXI para los aprendices del nuevo milenio en los países de la OCDE. Recuperado http://recursostic.educacion.es/blogs/europa/media/blogs/europa/informes/Habilidades y competencias siglo21 OCDE.pdf

Ortega, Juan Francisco y Ortega, José Ángel. (s.f.). Matemáticas: ¿Un problema de lenguaje? Recuperado el de http://urls.my/PxcXxX

Parra, Blanca. (1990). Dos concepciones de resolución de problemas. Revista Educación Matemática, 2(3), 22-32.

Pimm, David. (2002). El lenguaje matemático en el aula. Madrid: Ediciones Morata.

Programa Estado de la Nación (2013). Decimonoveno Informe Estado de la Nación en Desarrollo Humano Sostenible. San José, Costa Rica: Programa Estado de la Nación. Recuperado de http://www.estadonacion.or.cr/estado-nacion/informeactual?highlight=YTo0OntpOjA7czo3OiLDrW5kaWNIljtpOjE7czoxMDoiZGVzYXJyb2x sbyl7aToyO3M6Njoic29jaWFslitpOjM7czoxNzoiZGVzYXJyb2xsbyBzb2NpYWwiO30=

Quesada, Daniel. (1991)¿Es la Matemática un lenguaje? Revista de Filosofía, 3ª́ Época, 4(5), 31-43. Editorial Complutense, Madrid. Recuperado de http://revistas.ucm.es/index.php/RESF/article/viewFile/RESF9191120031A/11982

Radford, Luis. (2004). Semiótica cultural y cognición. Recuperado de http://www.cimm.ucr.ac.cr/ojs/index.php/eudoxus/article/viewFile/350/353

Ramos, Eduardo. (s.f.). Tesina sobre el lenguaje (Anatomía, evolución, teorías, lenguaje y pensamiento, glosario). Recuperado http://ideasapiens.blogsmedia.com/psicologia/educacion/tesina\%20lenguaje.htm

Real Academia Española de la Lengua. (2001). Diccionario de la Lengua Española. Recuperado de http://lema.rae.es/drae/?val=contexto

Rigo, Mirela, Páez, David y Gómez, Bernardo. (2009). Procesos Meta-Cognitivos en las clases de matemática elemental. Propuesta de un marco interpretativo. En María José González, María Teresa González y Jesús Murillo (Eds.), Investigación en 
educación Matemática XIII (pp. 435-444). Santander: SEIEM. Recuperado de http://www.seiem.es/publicaciones/archivospublicaciones/actas/Actas13SEIEM/SEIE MXIII Indice.pdf

Ruiz, Ángel y Chavarría, Jesennia. (2003). Educación matemática: escenario histórico internacional y construcción de una nueva disciplina. Revista UNICIENCIA 20(2), 1127. Facultad de Ciencias Exactas y Naturales. Universidad Nacional. Heredia, Costa Rica.

Schoenfeld, Alan. (1992). Learning to think mathematically: Problem solving, metacognition, and sense-making in Mathematics. Recuperado de http://www.cimm.ucr.ac.cr/ojs/index.php/eudoxus/article/viewFile/352/355

Tall, David. (1992). Students' Difficulties in Calculus. Plenary presentation in Working Group 3, ICME. Quebec. Canada. Recuperado de http://homepages.warwick.ac.uk/staff/David.Tall/pdfs/dot1993k-calculus-wg3-icme.pdf

Vigotsky, Lev. (2005). Pensamiento y lenguaje. La Habana: Editorial Pueblo y Educación. 